\title{
IMMUNOMODULATORY EFFECTS OF PAEONIFLORIN COMBINED TO SOLUBLE EGG ANTIGENS IN SCHISTOSOMIASIS MANSONI MURINE MODEL
}

\author{
By \\ NAGLAA FATHY ABD EL-AAL ${ }^{1 *}$ and EMAN HASSAN ABD ELBARY ${ }^{2}$ \\ Department of Medical Parasitology ${ }^{1}$, and Department of Pathology ${ }^{2}$, \\ Faculty of Medicine, Zagazig University, Egypt \\ ( ${ }^{*}$ Correspondence:naglaa_fathy220@yahoo.com),mobile 01097120886

\section{Abstract}

Praziquantel, the main schistosomiasis chemotherapy; cannot fully counteract the infection associated morbidity including hepatic fibrosis and its sequelae. A novel therapeutic strategy is mandatory. The current study is a novel trial to assess the immunomodulatory effect of Paeoniflorin (PAE) in combination to soluble egg antigen (SEA) in schistosomiasis mansoni murine model through; parasitological, histopathological, immunohistochemical and serological studies aiming to synergistic effect that not only eliminate the parasite but also ameliorate morbidity associated to infection. Forty laboratory bred Swiss albino male CD-1 mice were used. The mice were classified into five groups ( 8 mice each), control healthy, control infected, PAE treated (50 $\mathrm{mg} / \mathrm{kg} / \mathrm{d}$ ), SEA vaccinated $(50 \mu$ protein) and combined (PAE+SEA) groups. All mice groups were sacrificed 10 weeks post infection. Our results showed marked decrease in egg count/gm stool, worm burden, granuloma diameter, tissue transforming growth factor-beta1 (TGF- $\beta 1$ ) and serum interleukin-13 (IL-13) associated with marked increase in the serum interferon-gamma (INF- $\gamma$ ) level in (PAE+SEA) combined group compared to all groups.

Keywords: Schistosoma mansoni, Fibrosis, Cirrhosis, PZQ, Cytokines, IL-13, INF- $\gamma$, TGF- $\beta 1$.

\section{Introduction}

Schistosomiasis is still a complicated long standing chronic disease with serious morbidity worldwide (Gouveia et al, 2019). Praziquantel (PZQ) monotherapy still has certain precautions in schistosomiasis treatment, not only due to lack of its effectiveness against immature stages but also, cannot fully counteract the infection associated morbidity and its sequelae including periportal fibrosis, esophageal varices. Several studies depend on the combinations of other chemotherapies with PZQ to overcome limitations of PZQ monotherapy and for discovery of recent alternatives were already done (Abd El-Aal et al, 2017a; Hegazy et al, 2018; Gouveia et al, 2018). Abd El-Aal et al. (2017b) reported that combination of soluble egg antigen (SEA) and PZQ had a hopeful prophylactic anti-schistosomiasis mansoni especially during re-infection in endemic areas.

Paeoniflorin (PAE) is a powerful antiinflammatory and immune regulatory Chinase herbal therapy (Tu et al, 2019). Abd ElAal et al. $(2017 \mathrm{c})$ reported that PAE could be a potential chemotherapy against schistosoma mansoni and exceeded PZQ in targeting apoptosis and improving hepatic fibrosis.

During treatment of infectious disease, the immune modulation might be accompanied with shifts in some cytokine profiles balance; therefore help in control morbidity (Powell and Sonnenfeld, 2006). The search for alternative control measures to decrease schistosomiasis morbidity is mandatory. Hence, there were in need for new policies, targeting not only the parasite but also the long standing pathogenesis and chronicity.

This study is a novel trial to assess the immunomodulatory effect of PAE in combination to SEA on schistosomiasis mansoni murine model through; parasitological, histopathological, immunohistochemical and serological studies aiming to achieve better bioavailability and higher anti-inflammatory immune regulatory effects to counteract the schistosomiasis morbidity.

\section{Materials and Methods}

The current experimental study was performed from July 2018 to March 2019. 
Experimental mice: Forty Swiss Albino male laboratory bred CD-1 mice 6-8 weeks (ws) old (18-20g) were obtained from Schistosome Biological Supply Program (SBSP), TBRI. The mice were preserved on a standard commercial pelleted diet, available water and housing in an air conditioned room (22$26^{\circ} \mathrm{C}$ ) till the study end. They were infected subcutaneously with Egyptian strain of $S$. mansoni \pm 100 cercariae (Peters and Warren, 1969) that was purchased from TBRI infected Biomphalaria alexandrina snails.

Chemotherapy and vaccine: Paeoniflorin (PAE) (purity >98\%, P0038\#, Sigma-Aldrich, St. Louis, USA) was dissolved in $1 \%$ carboxymethyl cellulose and given orally $(50 \mathrm{mg} / \mathrm{kg}$ / day) $6 \mathrm{ws}$ post infection (pi) for one month (Abd El-Aal et al, 2017c).

Soluble egg antigen (SEA) was prepared at TBRI. SEA total protein content was determined according to Bradford (1976). Vaccination schedule was achieved (Nabih and Soliman, 1986). 200 $\mu$ SEA initial dose was given to mice with total antigen concentration contained $30 \mu \mathrm{g}$ proteins, $200 \mu \mathrm{l}$ boos-ter dose containing $20 \mu \mathrm{g}$ proteins was given two weeks after; thus, each mouse received $50 \mu \mathrm{g}$ as a total antigen dose of protein (Etewa et al, 2014).

Experimental design and treatment protocol: Forty Swiss albino male laboratory bred CD-1 mice were divided into five groups ( 8 mice each): Control healthy $(\mathrm{CH})$ non infected, non-treated, non-vaccinated mice; Control infected (CI): infected non treated, non-vaccinated mice; PAE treated: infected mice then treated with PAE; SEA vaccinated: vaccinated mice with SEA (50ug) then infected; PAE+SEA combined: vaccinated, infected then treated.

Treatment schedule was started 6ws/pi for a month, $1 \%$ carboxymethyl cellulose transporter orally for both $\mathrm{CH}$ and $\mathrm{CI}$ groups for one month. SEA was given initial dose then booster dose of SEA was given two weeks after. All mice groups were sacrificed by rapid decapitation under anesthesia $10 \mathrm{ws} / \mathrm{pi}$.

The results were evaluated as follows:
Parasitological study: A- Egg count per gram stool was done for each mouse by Kato thick smear method (Katz et al, 1972) started 6ws/pi to ensure the infection and as a follow up. B- Worm burden were recovered by liver and intestinal perfusion (Duvall and Dewitt, 1967). Reduction percentage of worm burden (R\%) was calculated (Tendler et al, 1986).

Histopathological study: The liver tissues were removed from all sacrificed mice, fixed in $10 \%$ formalin solution immediately then paraffin wax. Sections were stained with Hematoxylin and Eosin (H\&E) and with Masson's Trichrome stain to detect the histopathological changes and measure the mean granuloma diameter $(\mu \mathrm{m})$. The percent reduction in granuloma diameter was calculated (Bancroft and Stevens, 1982).

Immunohistochemical study: $5 \mu \mathrm{m}$ thick paraffin embedded hepatic sections from all studied groups were stained using AvidinBiotin Peroxidase technique for TGF- $\beta 1$ staining [antibody (2Ar2) ab64715]; Abcam, Cambridge, MA, USA. For scoring, 10 random fields of intralobular and periportal areas were evaluated under microscope at $40 \mathrm{X}$ magnification. The integral light density was determined by multimedia color pathographic (Chen et al, 2008).

Serological study: Blood samples from all studied mice groups were collected then centrifuged at $3000 \mathrm{rpm}$ for $15 \mathrm{~min}$, sera were separated and stored at $-80^{\circ} \mathrm{C}$ for assessment of serum levels of INF- $\gamma(\mathrm{ng} / \mathrm{ml})$ and IL-13 $(\mathrm{pg} / \mathrm{ml})$ using an enzyme linked immunosorbent assays (ELISA) kit (RAB0477 \& RAB0257 Sigma-Aldrich, St. Louis, USA, respectively) according to the manufacturer's instructions.

Ethical consideration: This experimental study was performed according to International guidelines approved by Research Ethics Committee, Faculty of Medicine, Zagazig University.

Statistical analysis: Data were computerized and statistically analyzed using SPSS program (Statistical Package for Social Sci- 
ence) version 18.0. Quantitative data were expressed as $\mathrm{M} \pm \mathrm{SD}$ (Standard deviation). Groups were compared using ANOVA test or Student's t-test. $\mathrm{P}$ value $<0.05$ indicates statistical significant, $\mathrm{P}$ value $<0.001$ indicates statistical high significant.

\section{Results}

There was a statistical significant reduction in egg count/gm stool 96.7\%, 84.2\% $41.7 \%$ in PAE+SEA, PAE and SEA groups compared to control infected non treated group $(\mathrm{CI})$. There was a statistical significant reduction in hepatic worm burden in PAE+SEA group $(96.4 \%)$ that exceeded PAE $(82.7 \%)$ and SEA $(47.6 \%)$ groups compared to CI.

Regarding granuloma diameter, there was statistical significant reduction $69.5 \%$, $30.1 \%$ 85.5\% in PAE, SEA and PAE+SEA groups respectively compared to CI (Tab. 1). Hepatic sections stained with H\&E showed granulomas in CI mice group were large hepatic fibrocellular granulomas with eosinophils, neutrophils, and lymphocytes and with central living ova. While, in the PAE \& PAE+SEA groups fibrocellular granulomas formed of degenerated ova surrounded by giant cells, pigmented macrophages, lymphocytes, plasma cells and fibrous tissue (Fig. 1A).

Hepatic sections stained with Masson's Trichrome showed decrease in the severity of hepatic fibrosis in PAE and PAE+SEA groups compared to SEA and CI groups (Fig. 1B).

Regarding TGF- $\beta 1$ expression, there was significant reduction $66.7 \%, 26.6 \& 89.5 \%$ in PAE $(0.314 \pm 0.03)$, SEA $(0.691 \pm 0.02) \&$ PAE+SEA $(0.099 \pm 0.04)$ groups compared to CI group (0.942 \pm 0.08$)$ (Fig. 1C)

Serologically serum INF- $\gamma$ level, there were $32.4,270.3,103.8 \& 483.9$ folds increase in CI $(326.8 \pm 11.3)$, PAE treated (564.7 \pm 10.9$),(398.2 \pm 13.8)$, SEA vaccinated $(398.2 \pm 13.8) \quad \& \quad$ PAE+SEA combined $(778.3 \pm 14.2)$ groups respectively compared to $\mathrm{CH}$ group $(294.4 \pm 12.7)$. On the other hand, regarding serum IL-13 level there was statistical significant increase $603.7 \& 562.5$ folds in CI \& SEA groups respectively, while, there was statistically insignificant increase 59.9 \& 36.6 folds in PAE \& PAE+SEA groups respectively compared to $\mathrm{CH}$ group (Tab. 2).

Table 1: Parasitological results in all groups

\begin{tabular}{|l|c|c|c|c|c|c|}
\hline \multirow{2}{*}{$\begin{array}{l}\text { Parameters } \\
\text { Groups }\end{array}$} & \multicolumn{2}{|c|}{ Egg count/gm stool } & \multicolumn{2}{c|}{ Worm burden } & \multicolumn{2}{c|}{ Hepatic granuloma diameter $(\mu \mathrm{m})$} \\
\cline { 2 - 6 } & $\mathrm{M} \pm \mathrm{SD}$ & $\% \mathrm{R}$ & $\mathrm{M} \pm \mathrm{SD}$ & $\% \mathrm{R}$ & $\mathrm{M} \pm \mathrm{SD}$ & $\% \mathrm{R}$ \\
\hline CI & $714.3 \pm 10.6$ & - & $33.6 \pm 1.8$ & - & $342.7 \pm 0.96$ & - \\
\hline PAE & $114.8 \pm 5.4 *$ & $84.2 \%$ & $5.8 \pm 0.64 *$ & $82.7 \%$ & $104.6 \pm 0.87 *$ & $69.5 \%$ \\
\hline SEA & $416.3 \pm 6.7$ & $41.7 \%$ & $17.6 \pm 0.82$ & $47.6 \%$ & $239.4 \pm 0.099$ & $30.1 \%$ \\
\hline PAE+SEA & $23.4 \pm 4.8 * *$ & $96.7 \%$ & $1.2 \pm 0.71 * *$ & $96.4 \%$ & $49.8 \pm 0.52 * * \#$ & $85.5 \%$ \\
\hline
\end{tabular}

ANOVA test ${ }^{\#}(\mathrm{p}<0.001) \&$ Student t-test, $*(\mathrm{P}<0.05), * *(\mathrm{p}<0.001)$ compared with CI; CI: control infected; PAE: Paeoniflorin; SEA: Soluble egg antigen.

Table 2: Serum INF- $\gamma(\mathrm{ng} / \mathrm{ml})$ and IL-13 $(\mathrm{pg} / \mathrm{mL})$ in all studied groups

\begin{tabular}{|c|c|c|}
\hline Variable & INF- $\gamma(\mathrm{ng} / \mathrm{ml})$ & IL-13 $(\mathrm{pg} / \mathrm{ml})$ \\
\hline CH & $294.4 \pm 12.7$ & $29.7 \pm 15.3$ \\
\hline CI & $326.8 \pm 11.3$ & $633.4 \pm 12.8^{* * \#}$ \\
\hline PAE & $564.7 \pm 10.9 *$ & $89.6 \pm 13.2 *$ \\
\hline SEA & $398.2 \pm 13.8$ & $592.2 \pm 14.7 * *^{\#}$ \\
\hline PAE+SEA & $778.3 \pm 14.2 * * \#$ & $66.3 \pm 10.6$ \\
\hline
\end{tabular}

ANOVA test ${ }^{\#}(\mathrm{p}<0.001)$ \& Student $\mathrm{t}$-test, $*(\mathrm{P}<0.05), * *(\mathrm{p}<0.001)$ compared with compared with $\mathrm{CH} ; \mathrm{CH}$ : control healthy.

\section{Discussion}

The main complain in S. mansoni endemic areas is the recurrent infection with long standing chronicity, new alternatives should be discovered to control schistosomiasis morbidity and prevent re-infection. In the current study, a novel therapeutic strategy aiming to achieve synergistic and/or additive effect that not only eliminate the parasite but also ameliorate morbidity associated to infection. Murine model was more suitable for this study as they have a similar growth 
as human schistosomiasis with high homologization (Abdul-Ghani and Hassan, 2010).

In the current study, the treatment protocol 6/ws/pi by administration of PAE orally $(50 \mathrm{mg} / \mathrm{kg} /$ day) for one month ( $\mathrm{Ji}$ et al, 2016) as previously verified with respectable results (Abd El-Aal et al, 2017c). Also, PAE was used in combination with SEA. SEA was previously proved with potential results and vaccination schedule started one week before infection and booster dose 2/ws/pi (Etewa et al, 2014).

In this study, Kato-Katz method was used 6/ws/pi to confirm mice infection and follow up the study. Kato-Katz method is the diagnostic keystone, simple inexpensive technique, commonly used in endemic areas of S. mansoni (Teixeira et al, 2007).

Regarding the anti-parasitic efficiency of PAE and SEA, there was significant reduction in egg count/g stool in PEA treated group that exceeded SEA group compared to CI group, whereas PAE+SEA combined group had the highest effect in this aspect. This result highlights the expectation of worm burden in different studied groups.

Regarding PAE+SEA group, there was mar-ked reduction in worm burden which exceeded PAE group compared to CI group. However, reduction of worm burden in SEA group was the lowest. This is nearby to ElAhwany et al. (2012) who detected marked decrease in worm burden following to administration of SEA previous $S$. mansoni infection. This anti-parasitic efficacy of the PAE+SEA combined group may be attributed to hepatic shift and death of adult worms by PAE and potentiation of its action by SEA through obstructing the oviposition process via increasing the dead eggs. Furthermore, SEA contains a variety of antigens that cross react with cercariae antigens, schistosomules and adult worm antigens. Taken together, Woolhouse (1994) and Curwen et al. (2004) detected the same suggestion. Moreover, Abdel-Ghaffar et al. (2005) and Abd El-Aal et al. (2017b) reported that combination of SEA and PZQ was recom- mended as it provided various complementary consequences; reduction in egg induced pathology, minimal parenchymal changes and eradication of worms. Although, $\mathrm{Li}$ et al. (2009) recorded that PAE has no antiparasitic effect on worm burden in mice infected with S. japonicum. On the other hand, Abd El-Aal et al. (2017c) attributed PAE anti-parasitic efficacy to the differences in schistosome species, dosage and timing of PAE treatment that could disturb the adult worm tegument.

The granuloma formation and fibrosis is the cornerstone of morbidity and mortality in schistosomiasis mansoni. The present results recorded significant reduction in granuloma diameter and fibrotic areas in PAE+ SEA group which was superior to PAE and SEA groups compared to CI group; this is attributed to the combination of PAE+SEA achieve synergistic or additive effect for local inhibition of the inflammatory mediators released at the granulomatous reaction or decrease in fibrotic markers expressions via improvement of immune response and cytokines modulation. This agreed with Abd ElAal et al. (2017c). Moreover, Abd El-Aal and Abdelbary (2019) detected that PAE is predicted to be a potential therapy for chronic hepatic diseases associated with fibrosis and angiogenesis, hopeful in protecting from advanced long standing serious complications. Coming with, Chen et al. (2015) who reported that PAE reduced the size of S. japonicum egg granulomas and fibrosis scores.

Cytokines are important regulators of immune-inflammatory responses and play effective role in regulation of granuloma formation and fibrosis. Hoffmann et al. (2002) reported the alteration in T helper cytokine profiles during the formation of granulomatous reaction. Transforming growth factor-beta1 (TGF- $\beta 1$ ) is a fibrosis potentiation Th2 cytokine, also, a good indicator for hepatic fibrosis and evaluation, via activation and differentiation of Hepatic stellate cells (HSCs) that stimulates fibroblast proliferation (Lan and Chung, 2012). Besides, 
Liu et al. (2012) reported that interleukin-13 (IL-13) endorses TGF- $\beta 1$ receptors activation.

The current results showed marked decrease in the fibrotic marker expression; TGF- $\beta 1$ in PAE+SEA combined group that was superior to PAE and SEA groups compared to CI group. The results are coming along with Ji et al. (2016) who reported that PAE produce suppression of TGF- $\beta 1$ resulted in diminution of pulmonary fibrosis. Besides, Abd El-Aal et al, (2017c) recorded that PAE monotherapy has anti-fibrotic effect which exceed PZQ through downregulation of TGF- $\beta 1$ fibrotic marker.

Caldas (2008) detected that in early infection phase, expression of Th1 cytokines was predominant and Th2 cytokines appearance started with chronicity. IFN- $\gamma$ is Th1 cytokine, detected early at the starting of hepatic granulomatous reaction, by the time granulomas size becomes maximal and cytokines production declined (de Jesus et al, 2004). IL-13 is Th2 cytokine, starts to appear 6-8 ws/pi and responsible for fibrosis and chronicity (Pearce and MacDonald, 2002).

The present results detected marked increase in serum level of INF- $\gamma$ in PAE+SEA group that exceeded PAE and SEA groups compared to CI group. On the other hand, there was marked decrease in serum IL-13 level in PAE+SEA group that exceeded PAE and SEA groups compared to CI group. This agreed with Stadecker et al. (2004) who detected that mice infected with a single large dose of cercariae and then treated with PZQ established Th1 dominant response with elevated SEA induced IFN- $\gamma$.

On the other hand, infection with repeated small doses of cercariae developed Th2 IL-5 response (Farah et al, 2000). This may highlight our attention for the important role of SEA administration in endemic areas as a prophylaxis against recurrent infection.

Interleukin-13 (IL-13) is the important Th2 cytokine responsible for fibrosis (Fallon et al, 2000). Some Th1 mediators such as interferon-gamma (INF- $\gamma$ ), IL-12, tumor ne- crosis factor (TNF- $\alpha$ ) prevent production of excess IL-13 during S. mansoni infection (Miranda et al, 2013).

Taken together, Wan et al. (2017) detected that egg induced hepatic granuloma and fibrosis regression were due to decreased IL13 expressions. Similar to results of the previous studies, Chu et al. (2007) reported that IL-13 activated macrophages to secrete TGF- $\beta 1$ that initiated granuloma formation, HSC proliferation, collagen production and fibrosis. Also, Abd EL-Aal et al. (2017c) detected a close positive correlation between serum IL-13 and TGF- $\beta 1$ fibrotic marker expression. Also, Montesano et al. (1997) reported that IFN- $\gamma$ has important role during schistosoiasis mansoni, prevent the release of Schistosoma eggs by adult worms and thus reduces hepatic fibrosis during schistosomiasis chronic phase. Moreover, IFN- $\gamma$ has potential role linked to the balance of IL-13 mediated fibrosis (Hesse et al, 2001). Miranda et al, (2013) recorded the potent anti-parasitic activity of IFN- $\gamma$ via release of nitric oxide.

From the present results, it can gain insight into the anti-helminthic, antifibrotic and protective properties of PAE combined to SEA that leads to death of viable eggs and prevents more deposition, which is considered to be the activating stimulus for several pathways that altered Th1/ Th2 cytokines expression. Liang et al. (2011) and Attia et al. (2013) reported that the anti-fibrotic effect of PAE therapy inhibited the gene expression of both Th1 and Th2, which alternated the Th1/Th2 cytokines; decreased TGF- $\beta 1 \&$ IL-13 and increased INF- $\gamma$; finally led to hepatic fibrosis regression.

SEA as prophylaxis or adjuvant aggravates and potentiates the effect of PAE and is considered the key factor in improvement of immune response or could be a sort of primary infection that prevents the challenge one.

\section{Conclusions}

The results confirmed that combination of PAE and SEA showed marked improves in 
parasitological, histopathological, immunological parameters and has immunomodulatory effects associated with shift in the balance of some cytokine profiles. Consequently, this approach might be considered for prophylaxis or for use in regions with intense reinfection levels since the combination might block or retard parasite infection and morbidity development.

Conflict of interest: The authors declared neither have conflict of interest nor received fund.

\section{References}

Abd El-Aal, NF, Abdelbary, EH, 2019: Paeoniflorin in experimental BALB/c mansoniasis: A novel anti-angiogenic therapy. Exp. Parasitol. 197:85-92.

Abd El-Aal, NF, Hamza, RS, Magdy, M, 2017 a: Anti-angiogenic and antilymphangiogenic role of praziquantel and artemether in experimental mansoniasis. Acta Parasitol. 62, 4:708-16.

Abd El-Aal, NF, Mohammed, FA, Magdy, M, 2017b: Immunoprotection of soluble egg antigens and praziquantel in challenged murine schistosomiasis mansoni. J. Egypt. Soc. Parasitol. 47, 1:1-12.

Abd El-Aal, NF, Hamza, RS, Harb, O, 2017c: Paeoniflorin targets apoptosis and ameliorates fibrosis in murine schistosomiasis mansoni: A novel insight. Exp. Parasitol. 183:23-32.

Abdel-Ghaffar, O, Rawi, SM, Ishaq, AI, 2005: Evaluation of the curative efficacy of RO 15-8843 against mansonian schistosomiasis in albino mice. J. Egypt Ger. Soc. Zool. 47:15-22.

Abdul-Ghani, RA, Hassan, AA, 2010: Murine schistosomiasis as a model for human schistosomiasis mansoni: similarities and discrepancies. Parasitol. Res. 107:1-8.

Attia, YM, Elalkamy, EF, Hammam, OA, Mahmoud, SS, El-Khatib, AS, 2013: Telmisartan, an AT1 receptor blocker and a PPAR gamma activator, alleviates liver fibrosis induced experimentally by Schistosoma mansoni infection. Parasites Vectors 6:199. http://www. parasitesandvectors.com/content/6/1/199.

Bancroft, S, Stevens, A, 1982: Theory and Practice of Histological Techniques. $2^{\text {nd }}$ Edition. Churchill-Livingston, New York.

Bradford, MM, 1976: A rapid and sensitive method for the quantitation of microgram qualities of protein utilizing the principle of protein dye binding. Anal. Biochem. 72:248-54.
Caldas, IR, Campi-Azevedo, AC, Oliveira, LF, Silveira, AM, Oliveira, RC, et al, 2008: Human schistosomiasis mansoni: immune responses during acute and chronic phases of the infection. Acta Trop. 108:109-17.

Chen, H, He, YW, Liu, WQ, Zhang, JH, 2008: Rosiglitazone prevents murine hepatic fibrosis induced by Schistosoma japonicum. World J. Gastroenterol. 14: 2905-11.

Chen, SR, Chen, XP, Lu, JJ, Wang, Y, Wang, YT, 2015: Potent natural products and herbal medici-nes for treating liver fibrosis. Chin. Med. 10: 7. https://doi.org/10.1186/s13020-015-0036y.

Chu, D, Luo, Q, Li, C, Gao, Y, Yu, L, et al, 2007: Paeoniflorin inhibits TGF- $\beta 1$ mediated collagen production by Schistosoma japonicum soluble egg antigen in vitro. Parasitology 134: 1611-21.

Curwen, RS, Ashton, PD, Johnston, DA, Wilson, RA, 2004: The Schistosoma mansoni soluble proteome: a comparison across four life cycle stages. Mol. Biochem. Parasitol. 138:57-66. de Jesus, AR, Magalhaes, A, Miranda, DG, Miranda, RG, Araujo, MI, et al, 2004: Association of type 2 cytokines with hepatic fibrosis in human Schistosoma mansoni infection. Infect. Immun. 72, 6:3391-7.

Duvall, RH, Dewitt, WB, 1967: An improved perfusion technique for recovering adult Schistosoma from laboratory animals. Am. J. Trop. Med. Hyg. 16, 4:483-4.

El-Ahwany, E, Bauiomy, IR, Nagy, F, Zalat, R, Mahmoud, O, et al, 2012: Regulatory cell responses to immunization with a soluble egg antigen in Schistosoma mansoni infected mice. Korean J. Parasitol. 50, 1:29-35.

Etewa, SE, Abd El-Aal, NF, Abdel-Rahman, SA, El-Shafey, MA, 2014: Parasitological evaluation of potential candidate vaccines in Schistosoma mansoni-infected mice. J. Parasitol. Ve-ct. Biol. 6, 2:23-30.

Fallon, PG, Richardson, EJ, Mckenzie, GJ, Mckenzie, AN, 2000: Schistosome infection of transgenic mice defines distinct and contrasting pathogenic roles for IL-4 and IL-13: IL-13 is a profibrotic agent. J. Immunol. 164:2585-91.

Farah, IO, Johansson, M, Loēvgren-Bengtson, K, Hau, J, 2000: Schistosoma mansoni in mice: the pattern of primary cercarial exposure determines whether a secondary infection postchemotherapy elicits a $\mathrm{T}$ helper- 1 or a $\mathrm{T}$ helper- 2 associated immune response. Scand. J. Immu- 
nol. 51:237-43.

Gouveia, MJ, Brindley, PJ, Gärtner, F, José, M, Correia da Costa, JM, et al, 2019: Combination anthelmintic/antioxidant activity against Schistosoma mansoni. Biomolecules 9:54; doi: 10.3390/ biom9020054

Gouveia, MJ, Brindley, PJ, Gärtner, F, José, M, Correia da Costa, JM, et al, 2018: Drug repur-posing for schistosomiasis combinations of drugs or biomolecules. Pharmaceut. 11:5. doi: 10.3390/ph11010015

Hegazy, LAM, Al Motiam, MH, Abd El-Aal, NF, Ibrahim, SM, Mohamed, HK, 2018: Evaluation of artesunate and praziquantel combination therapy in murine schistosomiasis mansoni. Iran J. Parasitol. 13, 2:193-203.

Hesse, M, Modolell, M, Flamme, AC, Schito, M, Fuentes, JM, et al, 2001: Differential regulation of nitric oxide synthase-2 and arginase-1 by type 1/type 2 cytokines in vivo: granulomatous pathology is shaped by the pattern of Larginine metabolism. J. Immunol. 167:6533-44.

Hoffmann, A, Levchenko, A, Scott, ML, Baltimore, D, 2002: The IkappaB-NF-kappaB signaling module: Temporal control and selective gene activation. Science 298, 5596:1241-5.

Ji, Y, Dou, Y, Zhoa, Q, Zhang, J, Yang, Y, et $a l$, 2016: Paeoniflorin suppresses TGF- $\beta$ mediated epithelial mesenchymal transition in pulmonary fibrosis through a Smad-dependent pathway. Acta Pharmacol. Sin. 37:794-804.

Katz, N, Chaves, A, Pellegrino, J, 1972: A simple device for quantitative stools thick smear technique in schistosomiasis mansoni. Rev. Inst. Med. Trop. S Paulo 14:397-400.

Lan, HY, Chung, AC, 2012: TGF-b/Smad signaling in kidney disease. Semin Nephrol. 32, 3: 236-43.

Li, X., Shen, J, Zhong, Z, Wen, H, Luo, Q, Wei, W, 2009: Paeoniflorin: a monomer from traditional Chinese medical herb ameliorates Schistosoma japonicum egg induced hepatic fibrosis in mice. J. Parasitol. 95, 6:1520-4.

Liang, YJ, Luo, J, Yuan, Q, Zheng, D, Liu, Y P, et al, 2011: New insight into the antifibrotic effects of praziquantel on mice in infection with Schistosoma japonicum. PLoS One 6, 5:20247.

Liu, Y, Munker, S, Müllenbach, R, Weng, $H$ L, 2012: IL-13 signaling in liver fibrogenesis. Front. Immunol. 3:116-23.

Miranda, MA, Kuehn, CC, Cardoso, JFR, Oliveira, LGR, Magalaes, LG, et al, 2013: Imm- unomodulatory effect of the alkaloidic extract of Solanum lycocarpum fruits in mice infected with Schistosoma mansoni. Exp. Parasitol. 133:396402

Montesano, MA, Freeman Jr, GL, Secor, W E, Colley, DG, 1997: Immunoregulatory idiotypes stimulate $\mathrm{T}$ helper 1 cytokine responses in experimental Schistosoma mansoni infections. J. Immunol. 158:3800-4.

Nabih, I, Soliman, AM, 1986: Studies on fresh water snails, specific intermediate host for schistosomiasis. II. Isolation of total protein from native and irradiated snails. Cell Mol. Biol. 32:3157.

Pearce, EJ, MacDonald, AS, 2002: The immunobiology of schistosomiasis. Nat. Rev. Immunol. 2: 499-511.

Peters, AP, Warren, KS, 1969: A rapid method of infecting mice and other laboratory animals with Schistosoma mansoni subcutaneous injection. J. Parsitol. 55:558-63.

Powell, JM, Sonnenfeld, G, 2006: The effects of Dehydroepiandrosterone (DHEA) on in vitro spleen cell proliferation and cytokine production. J. Interferon Cytokine Res. 26:34-9.

Stadecker, MJ, Ashai, H, Finger, E, Hernandez, HJ, Rutitzky, LI, et al, 2004: The immunobiology of Th1 polarization in high pathology schistosoniasis. Immunol. Rev. 201:168-79.

Teixeira, CF, Neuhauss, E, Bem, R, Romanzini, J, Graeff-Teixeira, C, 2007: Detection of Schistosoma mansoni eggs in feces through their interaction with paramagnetic beads in a magnetic field. PLoS Negl. Trop. Dis. 1:e73.

Tendler, M, Pinto, RM, Oliveira, LA, Gebara, G, Katz, N, 1986: Schistosoma mansoni: Vaccinetion with adult worm antigens. Int. J. Parasitol. 16, 4:347-52.

Tu, J, Guo, Y, Hong, W, Fang, Y, Han, D, et al, 2019: The Regulatory effects of paeoniflorin and its derivative paeoniflorin-60-O-benzene sulfonate CP-25 on inflammation and immune diseases. Front. Pharmacol. 10:57. doi:10.3389/f phar.00057

Wan, C, Jin, F, Du, Y, Yang, K, Yao, L, et al, 2017: Genistein improves schistosomiasis liver granuloma and fibrosis via dampening NF-kB signaling in mice. Parasitol. Res. 116, 4:116574.

Woolhouse, ME, 1994: Immunoepidemiology of human schistosomes: Taking the theory into the field. Parasitol. Today 10, 5:196-202. 


\section{Explanation of figures}

Fig.1: Hepatic sections: A- H \& E, x100; B- Masson's Trichrome x100 \& C- TGF- $\beta 1$ immunohistochemistry x100. CI: control infected; PAE: Paeoniflorin; SEA: Soluble egg antigen.
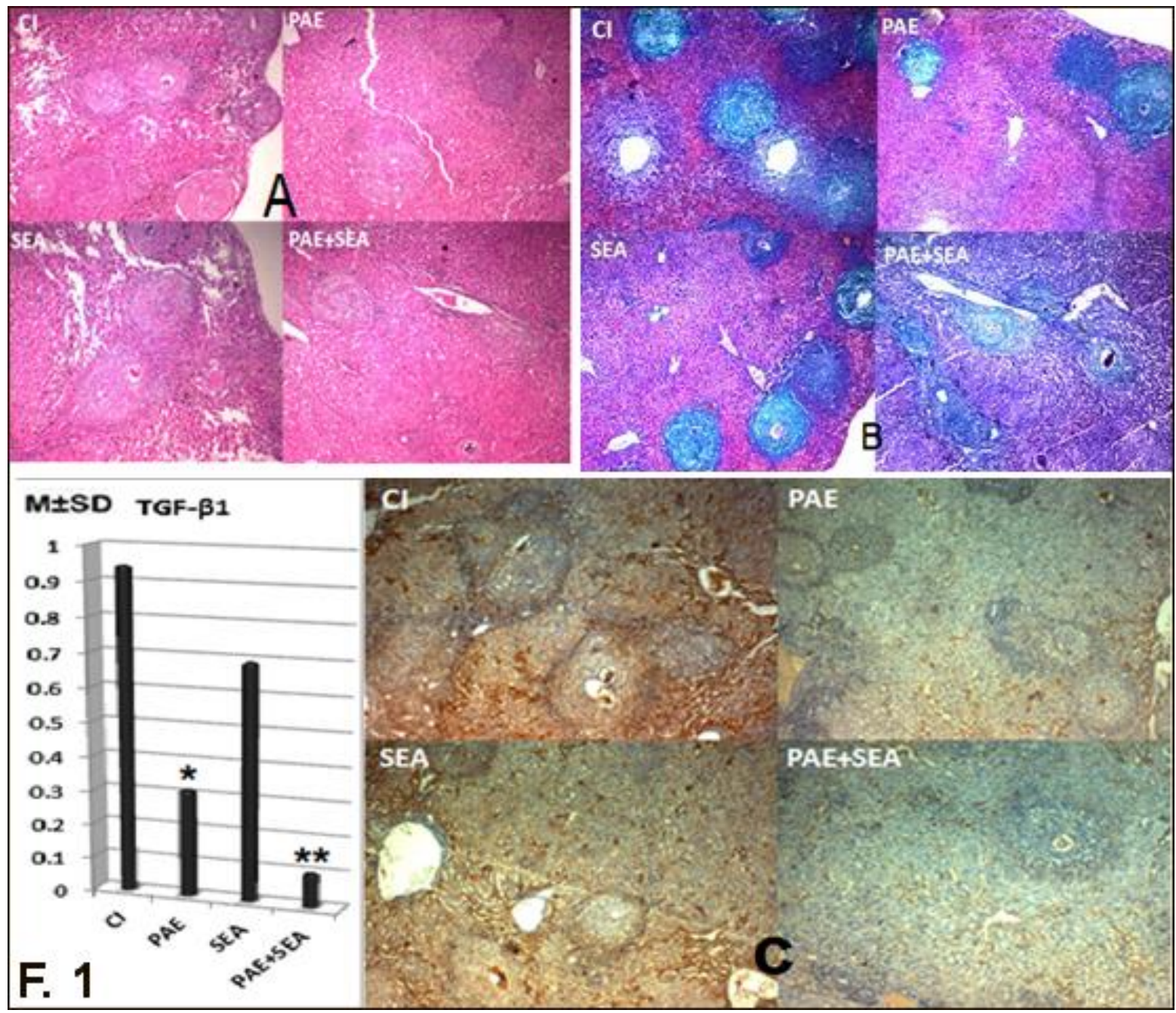